\title{
Myocardial function recovery interventional assessment and surgical pump removal
}

\author{
Johanna Mulzer ${ }^{1}$, Marcus Mueller ${ }^{1}$, Jan Knierim ${ }^{1}$, Pia Lanmueller ${ }^{1}$, Evgenij Potapov ${ }^{1,2}$ \\ ${ }^{1}$ Department of Cardiothoracic and Vascular Surgery, German Heart Center Berlin, Berlin, Germany; ${ }^{2}$ DZHK (German Center for Cardiovascular \\ Research), Partner Site Berlin, Berlin, Germany \\ Correspondence to: Dr. Johanna Mulzer, MD. Department of Cardiothoracic and Vascular Surgery, German Heart Center Berlin, Augustenburger \\ Platz 1, 13353 Berlin, Germany. Email: mulzer@dhzb.de.
}

Submitted Sep 11, 2020. Accepted for publication Apr 12, 2021.

doi: 10.21037/acs-2020-cfmcs-12

View this article at: http://dx.doi.org/10.21037/acs-2020-cfmcs-12

\section{Introduction}

The incidence of myocardial recovery on mechanical support is low $(1,2)$ and seen predominantly in younger patients with non-ischemic myopathy and shorter duration of heart failure before device implantation. Nevertheless, every outpatient visit should include screening for weaning candidates.

For assessment of myocardial recovery and weaning, a structured protocol $(3,4)$ is recommended to increase the probability of sustained myocardial recovery after explantation. For surgical pump removal, several options exist, including complete removal, partial removal and implantation of a specially designed recovery plug (5).

\section{Clinical vignette}

A 52-year-old male presented with cardiogenic shock due to cardiomyopathy of unknown origin and subsequently underwent implantation of a left ventricular assist device (LVAD) (Heartware HVAD, Framingham, MA, USA) as destination therapy, via median sternotomy. Six months later echocardiography showed a significantly improved biventricular ejection fraction. A reduction of a persistent severe mitral regurgitation was achieved by placing one Mitraclip (Abbott Laboratories, Abbott Park, IL, USA) 15 months after LVAD-implantation. Eighteen months postoperatively he was seen in the outpatient department in excellent clinical and psychological condition. His blood pressure was $90 / 52 \mathrm{mmHg}$. The electrocardiogram demonstrated sinus rhythm.

Echocardiography revealed normal function of the heart [left ventricular end-diastolic diameter (LVEDD) $51 \mathrm{~mm}$, left ventricular ejection fraction (LV-EF) $>55 \%$, right ventricular end-diastolic diameter (RVEDD) $32 \mathrm{~mm}$, right ventricular ejection fraction (RV-EF) 60\%], no more than mild valvular dysfunction.

Following our weaning protocol (3), we scheduled a pump stop echocardiography and a right heart catheterization with graft occlusion and pump stop (4).

As the echocardiography under LVAD-flow-reduction showed stable echo parameters, the pump was stopped for 5 minutes after extra anti-coagulation with 5,000 IE of heparin was given.

Two weeks later, a right heart catheterization took place under LVAD stoppage and occlusion of the outflow graft with a balloon catheter (Powerflex ProPTA, $12 \mathrm{~mm} / 2 \mathrm{~cm}$, Cordis, CA, USA). Pulmonary pressure was $28 / 13$ (mean 19) $\mathrm{mmHg}$, pulmonary capillary wedge pressure (PCWP) $8 \mathrm{mmHg}$ on-pump, and 32/16 (mean 21) $\mathrm{mmHg}$ and PCWP $10 \mathrm{mmHg}$ off-pump. The patient remained completely stable during the procedure. Four weeks later, the LVAD was scheduled for explantation.

\section{Surgical techniques}

At our institution, we prefer a specially designed titanium plug which allows complete removal from the left ventricle and enables the myocardium in the apical area to be preserved and therefore, cardio-pulmonary bypass (CPB) use can be avoided. In this case, the patient lies in supine position with rotation to the right side at approximately $45^{\circ}$. During off-pump explantation, CPB should be on stand- 
by, with both groins available for possible cannulation. The standard approach is via left anterior thoracotomy over the pump, mostly in the 6th intercostal space. Subsequently, exposition of the pump, the fixation mechanism and the first $2-3 \mathrm{~cm}$ of the outflow graft and cable is undertaken, followed by ligation and division of the graft and of the cable. Afterwards, off-pump removal of the pump, implantation and fixation of the recovery plug (Fittkau Metallbau GmbH, Berlin, Germany) was completed.

Subsequently, the cable and pericardial adaptation around the plug (if possible) were completely removed and the wound closed.

The patient was discharged 9 days later after an uneventful post-operative course.

\section{Comments}

The journey to cardiac recovery while on long-term mechanical cardiac support is still subject to many uncertainties. Clinical factors found to be of predictive value for positive outcomes are an acute presentation and a relatively shorter duration of heart failure, as well as a non-ischemic cause of cardiomyopathy (1). Patients who recovered sufficiently to allow explantation of their LVAD are prescribed to achieve cardiac and physical function capacities nearly equivalent to those of healthy controls (4). A protocol to identify candidates for potential explantation during routine outpatient visits includes echocardiographic imaging, pump stop echocardiography, right heart catheterizations and functional assessments, which collectively lead to good patient selection for sustained myocardial recovery (3).

When myocardial recovery occurs, there are several options:

(I) Complete removal, including the pump, fixation ring, outflow graft and driveline. This option requires over-sewing of the apex of the left ventricle and of the anastomosis to the aorta employing $\mathrm{CPB}$. The advantage lies in the complete removal of all components, especially if an LVAD-infection is present.

(II) Partial removal, with the pump and complete driveline being retrieved through a small incision above the pump in the sixth intercostal space. As this requires oversewing of the apex, this approach also requires $\mathrm{CPB}$.

(III) Percutaneous interventional ligation of the outflow graft through a small subcostal incision to stop backflow, with transection of the driveline below the skin while the outflow graft, pump and driveline remain in situ. This approach has a more palliative character and should not be used routinely.

(IV) Off-pump by using a specially designed recovery plug.

\section{Clinical results}

An analysis of all patients supported with LVAD and explanted for myocardial recovery at the German Heart Center $2008-2018(\mathrm{n}=58$, mean age $42.1 \pm 12.6$ years, 40 male, 18 female) showed that 44 patients were explanted by using the recovery plug, while 10 patients had apex direct closure and pump removal, and in 4 cases, the pump was left in situ. There were no cases of plug infection or thrombosis.

\section{Advantages}

The most elegant way to explant an LVAD for myocardial recovery is off-pump using a specially designed "recovery plug", as described in this article. This minimally invasive approach minimizes the surgical trauma and shortens the length of the procedure. Additionally, there is no need for anti-coagulation beyond 6 months.

If heart failure recurs and an LVAD is needed for a second time, the fixation ring may be re-used after removal of the plug (5).

\section{Caveats}

Although normal pre-explant exercise status, hemodynamic and echocardiographic function were important predictors for sustained myocardial recovery, the underlying pathology needs to be considered. Recurrence of heart failure is often in patients with chemotoxic or ischemic cardiomyopathy.

\section{Acknowledgments}

Funding: None.

\section{Footnote}

Conflicts of Interest: The authors have no conflicts of interest to declare.

Open Access Statement: This is an Open Access article distributed in accordance with the Creative Commons 
Attribution-NonCommercial-NoDerivs 4.0 International License (CC BY-NC-ND 4.0), which permits the noncommercial replication and distribution of the article with the strict proviso that no changes or edits are made and the original work is properly cited (including links to both the formal publication through the relevant DOI and the license). See: https://creativecommons.org/licenses/by-nc-nd/4.0/.

\section{References}

1. Antonides CFJ, Schoenrath F, de By TMMH, et al. Outcomes of patients after successful left ventricular assist device explantation: a EUROMACS study. ESC Heart Fail 2020;7:1085-94.

2. Potapov EV, Antonides C, Crespo-Leiro MG, et al. 2019

Cite this article as: Mulzer J, Mueller M, Knierim J, Lanmueller P, Potapov E. Myocardial function recovery interventional assessment and surgical pump removal. Ann Cardiothorac Surg 2021;10(3):402-404. doi: 10.21037/acs-2020cfmcs-12
EACTS Expert Consensus on long-term mechanical circulatory support. Eur J Cardiothorac Surg 2019;56:230-70.

3. Knierim J, Heck R, Pieri M, et al. Outcomes from a recovery protocol for patients with continuous-flow left ventricular assist devices. J Heart Lung Transplant 2019;38:440-8.

4. Jakovljevic DG, Yacoub MH, Schueler S, et al. Left ventricular assist device as a bridge to recovery for patients with advanced heart failure. J Am Coll Cardiol 2017;69:1924-33.

5. Potapov EV, Stepanenko A, Hennig E, et al. A titanium plug simplifies left ventricular assist device removal after myocardial recovery. J Heart Lung Transplant 2010;29:1316-7. 